J. DIFFERENTIAL GEOMETRY

103 (2016) 297-318

\title{
NORMAL FAMILY THEORY AND GAUSS CURVATURE ESTIMATE OF MINIMAL SURFACES IN $\mathbb{R}^{m}$
}

\author{
Xiaojun Liu \& Xuecheng Pang
}

\begin{abstract}
In this paper, we first extend Zalcman's principle of normality to the families of holomorphic mappings from Riemann surfaces to a compact Hermitian manifold. We then use this principle to derive an estimate for Gauss curvatures of the minimal surfaces in $\mathbb{R}^{m}$ whose Gauss maps satisfy some property $\mathcal{P}$, in the spirit of Bloch's heuristic principle in complex analysis. Consequently, we recover and simplify the known results about value distribution properties of the Gauss map of minimal surfaces in $\mathbb{R}^{m}$.
\end{abstract}

\section{Introduction}

According to the Picard's little theorem, a function meromorphic on the whole complex plane that omits three distinct values $a, b, c$ in $\mathbb{P}^{1}$ must be constant. On the other hand, Montel's theorem states that a family of functions meromorphic on a domain $G \subset \mathbb{C}$, each of which omits the fixed values a, $b, c$, must be normal. Based on this phenomenon, Bloch (see [1]) pointed out that a family of meromorphic functions which have a property $\mathcal{P}$ in common on a domain $D \subset \mathbb{C}$ should be normal on $D$ if the property $\mathcal{P}$ forces a meromorphic function on the whole complex plane $\mathbb{C}$ to be constant (this is known as the Bloch's heuristic principle). Based on this principle, Bloch was able to predict or conjecture several important results, such as Ahlfors's Five Islands theorem and Cartan's theorem on holomorphic curves omitting hyperplanes, among others. Later, Zalcman (see [26, 27]) formulated a more precise statement (known as Zalcman's principle and the Zalcman-Pang principle $[\mathbf{1 8}, \mathbf{1 9}])$ to dertermine the normality for families of meromorphic functions.

In the theory of minimal surfaces, there is a similar phenomenon. Every time, while there was a result about the complete minimal surfaces satisfying a certain condition being flat, there was always a corresponding result about the Gauss curvature estimate for the (noncomplete) minimal surfaces that satisfy the same condition. For example, while the well-known Bernstein theorem states that any minimal

Received 7/2/2013. 
graph $z=f(x, y)$ on $\mathbb{R}^{2}$ must be flat, Heinz's theorem (see [10]) gives a Gauss curvature estimate for the minimal graph $z=f(x, y)$ on a disc in $\mathbb{R}^{2}$. Fujimoto (see $[\mathbf{6}]$ ) proved that the complete minimal surfaces in $\mathbb{R}^{3}$ whose Gauss maps omit the given five distinct points of the sphere must be flat, while in the same paper, he also gave the Gauss curvature estimate for such (non-complete) minimal surfaces. There were also this type results involving stability: while Do Carmo and Peng, Fisher-Colbrie and Schoen (see [2, 5]), and Pogorelov (see [20]) proved independently that the only complete stable minimal surface in $\mathbb{R}^{3}$ is the plane, Schoen (see [24]) later proved the stronger result that the stability assumption for a non-complete minimal surface implies the curvature estimate $|K(p)| d(p)^{2} \leq C$, where $K$ is the Gauss curvature of the surface and $d(p)$ is the geodesic distance form $p$ to the boundary of the surface. In [21], Ros developed a unified approach to the study of these problems (i.e., the flatness and the Gauss curvature estimate of minimal surfaces), similar to the Bloch and Zalcman principle in complex analysis.

Being inspirited by Ros' paper, we study in this paper the normality of families of holomorphic mappings from Riemann surfaces (or more generally from the Kobayashi hyperbolic manifolds) into a compact Hermitian manifold, as well as the minimal surfaces in $\mathbb{R}^{m}$. We first extend Zalcman's principle and then use this principle to derive an estimate for Gauss curvatures of the minimal surfaces in $\mathbb{R}^{m}$ whose Gauss maps satisfy some property $\mathcal{P}$, in the spirit of Bloch's heuristic principle. As consequences, we recover results about value distribution properties of the Gauss map of minimal surfaces in $\mathbb{R}^{m}$. Finally, we present a simpler proof of Ru's result [22] on complete minimal surfaces in $\mathbb{R}^{m}$ whose Gauss map omits more than $\frac{m(m+1)}{2}$ hyperplanes in general position in $\mathbb{P}^{m-1}$.

Acknowledgments. Research supported by the NNSF of China (Grant No. 11371139) and the first author also supported by the NNSF of China (Grant No. 11401381).

\section{A principle for normality of the family of holomorphic mappings from Riemann surfaces into a compact Hermitian manifold}

In this section, we extend Zalcman's principle of normality to the families of holomorphic mappings from Riemann surfaces to a compact Hermitian complex manifold (see Theorem 2, below).

2.1. The notion of normal family and Marty's criterion. Let $M$ be a connected Hermitian manifold of dimension $m$ with Hermitian 
metric $h_{M}$, and let $N$ be a compact connected Hermitian manifold of dimension $s$ with Hermitian metric $h_{N}$. The space $\mathcal{C}(M, N)$ of continuous mappings between $M$ and $N$ endowed with the compact-open topology is second countable so that a metric can be furnished in $\mathcal{C}(M, N)$ with induces the compact-open topology. Note that a sequence $\left\{f_{n}\right\}$ in $\mathcal{C}(M, N)$ converges to $f$ in $\mathcal{C}(M, N)$ in this topology if and only if $f_{n}$ converges to $f$ uniformly on compact subset of $M$. The space $\mathcal{H}(M, N)$ of holomorphic mappings from $M$ into $N$ is a closed subspace of $\mathcal{C}(M, N)$.

Definition 2.1. A family $\mathcal{F} \subset \mathcal{H}(M, N)$ is called normal if every sequence of $\mathcal{F}$ contains a subsequence that is relatively compact in $\mathcal{H}(M, N)$, i.e., any sequence $f_{n} \in \mathcal{F}$ contains a subsequence that converges to $f \in \mathcal{H}(M, N)$ uniformly on every compact subset of $M$.

Definition 2.2. A family $\mathcal{F} \subset \mathcal{C}(M, N)$ is called equicontinuous if for every $\varepsilon>0$ and $p \in M$, there exists $\delta>0$, such that $d_{M}(p, q)<\delta$ implies $d_{N}(f(p), f(q))<\varepsilon$ for all $f \in \mathcal{F}$, where $d_{M}$, $d_{N}$ denotes the distance function on $N$ generated by $h_{M}, h_{N}$, respectively.

Remark 1. If $N$ is compact, then $\mathcal{F}$ is normal if and only if it is equicontinuous.

For $p \in M$ and $\xi \in T_{p}(M)$, let

$$
K_{M}(p, \xi)=\inf \left\{a>0, \exists f \in \mathcal{H}(D, M), f(0)=p, f^{\prime}(0) a=\xi\right\} .
$$

$K_{M}$ is called the infinitesimal Kobayashi pseudometric. The complex manifold $M$ is called (Kobayashi) hyperbolic if the Kobayashi pseudometric $K_{M}$ is a metric.

Remark 2. On the open disc $M:=D(r), K_{M}(z, \xi)=r|\xi|_{\text {euc }} /\left(r^{2}-\right.$ $|z|^{2}$ ), which coincides with the Poincare metric.

For every $f \in \mathcal{H}(M, N)$, let

$$
\Lambda_{f}(z)=\sup _{\|\xi\|=1} h_{N}(f(z), d f(z) \xi)
$$

and, assuming that $M$ is hyperbolic,

$$
Q_{f}(z)=\sup _{\|\xi\|=1} \frac{h_{N}(f(z), d f(z) \xi)}{K_{M}(z, \xi)}
$$

where $\|\xi\|$ is the norm with respect to the metric $h_{M}$. Note that $\Lambda_{f}(z)$ is the "maximal derivative" of $f$ at $z$ and $Q_{f}(z)$ is the "maximal derivative" of $f$ with respect to $K_{M}$ at $z . Q_{f}(z)$ is invariant under $\phi \in A u t(M)$ in the sense that $Q_{f \circ \phi}(z)=Q_{f}(\phi(z))$ for all $z \in M$. This follows from the invariant property of $K_{M}$. This invariant property for $Q_{f}(z)$ plays an important role.

We have the following result. 
Proposition 2.1 (Marty's Criterion). Let $M$ be hyperbolic and let $N$ be compact. Then a family $\mathcal{F} \subset \mathcal{H}(M, N)$ is normal if and only if the following equivalent statements hold: that

(i) For each compact $K \subset M$, there exists a constant $C(K)>0$, such

$$
\sup _{z \in K}\left\{Q_{f}(z), f \in \mathcal{F}\right\}<C(K) .
$$

(ii) For each compact $K \subset M$, there exists a constant $C(K)>0$, such that

$$
\sup _{z \in K}\left\{\Lambda_{f}(z), f \in \mathcal{F}\right\}<C(K)
$$

Proof. The equivalence of statements (i) and (ii) is obvious. So we need only to prove (i). Suppose that, for each compact $K \subset M$, there exists a constant $C(K)>0$, such that $\sup _{z \in K}\left\{Q_{f}(z), f \in \mathcal{F}\right\}<C(K)$. Then $\mathcal{F}$ is equicontinuous and, hence, normal from the compactness of $N$.

Conversely, suppose that $\mathcal{F}$ is normal but (i) fails. Then there must be a compact subset $K \subset M$, a sequence of points $p_{n} \in K$ and $p_{n} \rightarrow p_{0} \in$ $K$, and a sequence of functions $f_{n} \in \mathcal{F}$, such that $Q_{f_{n}}\left(p_{n}\right)>n$. Take a local coordinate $\left(p_{0}, U\right)$ for $M$ around $p_{0}$ with $\left.T(M)\right|_{U}=U \times \mathbf{C}^{m}$, and assume that $p_{n} \in U$ for $n>n_{0}$. Under the chosen coordinates, there is a sequence of unit vectors $\xi_{n} \in \mathbb{C}^{m}$ for which $\xi_{n} \rightarrow \xi_{0},\left|\xi_{0}\right|=1$ such that

$$
h_{N}\left(f_{n}\left(p_{n}\right), d f_{n}\left(p_{n}\right) \xi_{n}\right)>n K_{M}\left(p_{n}, \xi_{n}\right) .
$$

Since $\mathcal{F}$ is normal, there exists a subsequence of $\left\{f_{n}\right\}$ (which we still denote as $\left.\left\{f_{n}\right\}\right)$, such that $f_{n}$ converges to $f_{0} \in \mathcal{H}(M, N)$ uniformly on compact subsets of $M$. Since $N$ is compact, the left side of (3) tends to a finite number $h_{N}\left(f_{0}\left(p_{0}\right), d f_{0}\left(p_{0}\right) \xi_{0}\right)$, while the right-hand side, because of the hyperbolic of $M$, can be made as large as we want as $n \rightarrow \infty$. This is a contradiction.

q.e.d.

\subsection{A principle for normality of the family of holomorphic} mappings from Riemann surfaces into $\mathbb{P}^{s}(\mathbb{C})$. In this subsection, we establish a principle (similar to Zalcman's principle) for normality of the family of holomorphic mappings from Riemann surfaces into $\mathbb{P}^{s}(\mathbb{C})$. We remark that while it has been enough to serve our purpose, the results can be easily extended to families in $\mathcal{H}(M, N)$ for the general Hermitian manifolds $M$ and $N$, with $M$ being Kobayashi hyperbolic and $N$ being compact.

Denote by $\Sigma$ a (connected) Riemann surface, $\mathbb{C}$ the finite complex plane, $\mathbb{P}^{s}(\mathbb{C})$ the complex projective space of dimension $s$, and $D(r)=$ $\{z \in \mathbb{C}:|z|<r\}, \bar{D}(r)=\{z \in \mathbb{C}:|z| \leq r\}$ the open and closed Euclidean disc of radius $r>0$, respectively. The unit disc will be denoted 
by $D$. Denote by $\mathcal{H}(\Sigma)$ the set of holomorphic maps from $\Sigma$ to $\mathbb{P}^{s}(\mathbb{C})$. In this case, according to (1), if $\Sigma$ is hyperbolic, then for any $f \in \mathcal{H}(\Sigma)$,

$$
Q_{f}^{2}(z)=\frac{f^{*} \omega_{F S}}{\omega_{\Sigma}}
$$

where $\omega_{F S}$ is the Fubini-Study form on $\mathbb{P}^{s}(\mathbb{C})$ and $\omega_{\Sigma}$ is the (hyperbolic) metric form on $\Sigma$. In particular, for $f \in \mathcal{H}(D)$,

$$
Q_{f}^{2}(z)=\frac{d d^{c} \log \|f\|^{2}}{1 /\left(1-|z|^{2}\right)^{2} \sqrt{-1} d z \wedge d \bar{z}} .
$$

Let $\mathcal{P}$ be an arbitrary property for holomorphic maps; we put

$$
\mathcal{P}(\Sigma)=\{g \in \mathcal{H}(\Sigma): g \text { satisfies the property } \mathcal{P}\} .
$$

Given a property $\mathcal{P}$, we consider the following assertions:

I) For any two Riemann surfaces $\Sigma$ and $\Sigma_{1}$, and for any holomorphic map without branch points $\phi: \Sigma \rightarrow \Sigma_{1}$,

$$
\text { if } g \in \mathcal{P}\left(\Sigma_{1}\right) \text {, then } g \circ \phi \in \mathcal{P}(\Sigma) \text {. }
$$

II) Let $\Sigma$ be any Riemann surface and $g \in \mathcal{H}(\Sigma)$. If for any relatively compact domain $\Omega$ of $\Sigma$ one has

$$
\left.g\right|_{\Omega} \in \mathcal{P}(\Omega), \text { then } g \in \mathcal{P}(\Sigma)
$$

III) For any Riemann surface $\Sigma, \mathcal{P}(\Sigma)$ is a closed subset of $\mathcal{H}(\Sigma)$.

IV) For any Riemann surface $\Sigma, \mathcal{P}(\Sigma)$ is compact in $\mathcal{H}(\Sigma)$.

If $\mathcal{P}$ satisfies the axioms I), II), and III), we will say that it is a closed property, and if $\mathcal{P}$ satisfies the axioms I), II), and IV), we will say that it is a compact property.

Remark 3. (i) It is obvious that compact property implies closed property. (ii) We also note that if $\mathcal{P}$ is a property such that $\mathcal{P}(\Sigma)$ is compact in $\mathcal{H}(\Sigma)$, then every family $\mathcal{F} \subset \mathcal{H}(\Sigma)$ satisfying $\mathcal{P}$ must be normal. For this reason, in what follows, we only need to seek the criterions for compact property.

Lemma 2.2. Let $\mathcal{P}$ be a compact property; then $\mathcal{P}(\mathbb{C})$ contains only constant maps.

Proof. Let $g \in \mathcal{P}(\mathbb{C})$, and if this is not the case, let there exist two distinct points $z_{1}$ and $z_{2} \in \mathbb{C}$, such that $g\left(z_{1}\right) \neq g\left(z_{2}\right)$. Define the sequence $g^{(n)}: \mathbb{C} \rightarrow \mathbb{P}^{s}(\mathbb{C}), n=1,2, \ldots$, by $g^{(n)}(z)=g(n z)$, for any $z \in \mathbb{C}$. From I) we see that $g^{(n)}$ lies in $\mathcal{P}(\mathbb{C})$. So, by IV), we have $\left\{g^{(n)}\right\}$ locally uniformly converges to some $G \in \mathcal{H}(\mathbb{C})$. Then $\lim _{n \rightarrow \infty} g^{(n)}\left(z_{1} / n\right)=$ $\lim _{n \rightarrow \infty} g^{(n)}\left(z_{2} / n\right)=G(0)$, which contradicts $g^{(n)}\left(z_{1} / n\right)=g\left(z_{1}\right) \neq g\left(z_{2}\right)=$ $g^{(n)}\left(z_{2} / n\right)$. Thus, $g$ is constant.

q.e.d.

Lemma 2.3. Let $\mathcal{P}$ be a closed property. Then the following assertions are equivalent: 
i) $\mathcal{P}$ is a compact property.

ii) $\mathcal{P}(D)$ is relatively compact.

Proof. If $\mathcal{P}$ is a compact property, by axiom IV), $\mathcal{P}(D)$ is relatively compact. Conversely, for any Riemann surface $\Sigma$, a family of holomorphic maps over $\Sigma$ is relatively compact if and only if its restriction to a neighborhood of each point of it is relatively compact. Then, from axioms I) and III), we have $\mathcal{P}$ is a compact property.

q.e.d.

Theorem 1 (An extension of Zalcman's principle for normality). Let $\mathcal{P}$ be a closed property; then the following two assertions are alternative.

i) $\mathcal{P}$ is a compact property. Or:

ii) There exists $g \in \mathcal{P}(\mathbb{C})$, such that $\Lambda_{g}(0)=1$ and $\Lambda_{g}(\zeta) \leq 1$, for all $\zeta \in \mathbb{C}$.

Proof. Suppose that $\mathcal{P}$ is not compact. By Lemma 2.3, $\mathcal{P}(D)$ is not relatively compact. By Proposition 2.1, there are sequences $\left\{f_{n}\right\} \in \mathcal{P}(D)$ and $\left\{z_{n}\right\} \subset D$ such that $Q_{f_{n}}\left(z_{n}\right) \rightarrow \infty$. By the invariance property of $Q_{f_{n}}(z)$ under $\operatorname{Aut}(D)$, we can assume that $z_{n}=0$ for all $n$. From I) we have that the maps $F_{n}(z): D \rightarrow \mathbb{P}^{s}(\mathbb{C})$, given by $F_{n}(z):=f_{n}(z / 2)$, are in $\mathcal{P}(D)$. Moreover, $Q_{F_{n}}(0) \rightarrow+\infty$, and, by definition, $Q_{F_{n}}=0$ on $\partial D$ for every $n$. So, composing if necessary with conformal transformations of $D$, we can assume that, for every $n$,

$$
\max _{z \in D} Q_{F_{n}}(z)=Q_{F_{n}}(0) \text {. }
$$

Let $R_{n}:=Q_{F_{n}}(0)$, and notice that $\Lambda_{F_{n}}(z)=\frac{1}{1-|z|^{2}} Q_{F_{n}}(z)$; then we get

$$
\Lambda_{F_{n}}(0)=R_{n} \rightarrow+\infty, \text { and } \Lambda_{F_{n}}(z) \leq \frac{R_{n}}{1-|z|^{2}}
$$

for any $z \in D$ and any $n$. We define a sequence of mappings $g_{n}$ : $D\left(R_{n}\right) \rightarrow \mathbb{P}^{s}(\mathbb{C})$, by $g_{n}(z)=F_{n}\left(z / R_{n}\right)$ for each $z_{n} \in D\left(R_{n}\right)$. Then $g_{n}$ are in $\mathcal{P}\left(D\left(R_{n}\right)\right)$,

$$
\Lambda_{g_{n}}(0)=1, \text { and } \Lambda_{g_{n}}(z) \leq\left(1-\frac{|z|^{2}}{R_{n}^{2}}\right)^{-1},
$$

for any $z \in D\left(R_{n}\right)$ and $n$. So $\Lambda_{g_{n}}$ is uniformly bounded on compact subsets of $\mathbb{C}$, and then, by taking a subsequence if necessary, we can assume that $g_{n}$ converges to a map $g: \mathbb{C} \rightarrow \mathbb{P}^{s}(\mathbb{C})$. As $\mathcal{P}$ is closed, using III), we see that $\left.g\right|_{D(r)} \in \mathcal{P}(D(r))$, and from II), we have that $g \in \mathcal{P}(\mathbb{C})$. Finally, from the properties of $g_{n}$, we conclude that $\Lambda_{g}(0)=1$ and $\Lambda_{g}(\zeta) \leq 1$, for all $\zeta \in \mathbb{C}$. This finishes the proof. q.e.d.

Below, we give some concrete examples of compact properties. 
Example 1. Let $H(q)=\left\{H_{j}\right\}_{j=1}^{q}$ be a collection of hyperplanes in $\mathbb{P}^{s}(\mathbb{C})$ located in general position. We consider the property $\mathcal{P}_{H(q)}$ defined for any Riemann surface $\Sigma$ by

$$
\begin{aligned}
\mathcal{P}_{H(q)}(\Sigma)= & \left\{g \in \mathcal{H}(\Sigma): \text { either } g(\Sigma) \cap H_{j}=\emptyset \text { or } g(\Sigma) \subset H_{j}\right. \\
& \text { for every } 1 \leq j \leq q\} \cup\{\text { constant maps }\} .
\end{aligned}
$$

We claim that if $q \geq 2 s+1$, then $\mathcal{P}_{H(q)}$ is a compact property.

To prove the claim, first notice that $\mathcal{P}_{H(q)}$ satisfies trivially the axioms I) and II). By Hurwitz's theorem, axiom III) also holds, i.e., $\mathcal{P}_{H(q)}(\Sigma)$ is a closed subset of $\mathcal{H}(\Sigma)$. To check axiom IV), from Theorem 1 , we only need to show that if $g: \mathbb{C} \rightarrow \mathbb{P}^{s}$ is holomorphic such that either $g(\mathbb{C}) \subset H_{j}$ or $g(\mathbb{C}) \cap H_{j}=\emptyset$ for every $1 \leq j \leq q$ with $q \geq 2 s+1$, then $g$ must be constant (so (ii) in Theorem 1 does not hold, since $\Lambda_{g} \equiv 0$ if $g$ is constant, and thus (i) must hold, i.e., $\mathcal{P}_{H(q)}$ is a compact property). To prove our statement, denote by $J \subset\{1, \ldots, q\}$ such that $j \in J$ if and only if $g(\mathbb{C}) \subset H_{j}$, and let $X_{J}:=\bigcap_{j \in J} H_{j}$. We can identify $X_{J}$ with a project space of dimension $s-k$, where $k=\# J$. Notice that the restrictions of $H_{j}, j \notin J$, to $X_{J}$ are hyperplanes which are still in general position in $X_{J}$ (this is immediate from the definition). Note that by $q-k \geq 2 s+1-k \geq 2(s-k)+1$, and by the results of Green $[\mathbf{9}]$ and Fujimoto [8], $g$ must be constant, which proves our statement.

Note that, by the result of Eremenko and Sodin (see [4]) or Noguchi and Winkelmann (see [14]), the above result also holds for hypersurfaces in general position.

Another important concrete example is for holomorphic maps intersecting hyperplanes with high multiplicities. We first recall the following.

Definition 2.3. Let $f: \Sigma \rightarrow \mathbb{P}^{s}(\mathbb{C})$ be a holomorphic map. Let $p \in$ $\Sigma$. A local reduced representation of $f$ around $p$ is a holomorphic map $\mathbf{f}: U \rightarrow \mathbb{C}^{s+1}-\{0\}$, such that $\mathbb{P}(\mathbf{f})=f$, where $U$ is a neighborhood of $p$ and $\mathbb{P}$ is the projection map of $\mathbb{C}^{s+1}-\{0\}$ onto $\mathbb{P}^{s}(\mathbb{C})$.

Definition 2.4. Let $\mu>0$ is an integer. The holomorphic map $f$ : $\Sigma \rightarrow \mathbb{P}^{s}(\mathbb{C})$ is said to be ramified over a hyperplane $H=\left\{[w] \in \mathbb{P}^{s}(\mathbb{C}):<\right.$ $\mathbf{a}, w>=0\}$ with multiplicity at least $\mu$ if all zeros of $<\mathbf{f}, \mathbf{a}>$ have orders at least $\mu$, where $\mathbf{f}$ is a local reduced representation of $f$ (it is easy to check that this definition is independent of the choices of the reduced representations). If either the image of $f$ completely omits $H$ or $f(\Sigma) \subset H$, we shall say that $f$ is ramified over $H$ with multiplicity $\infty$.

The following result of Nochka (see [13, Theorem 4]) extends the result of Green and Fujimoto.

Proposition 2.4 (Nochka's Theorem). Suppose that $H_{1}, \ldots, H_{q}$ are $q \geq 2 s+1$ hyperplanes in $\mathbb{P}^{s}(\mathbb{C})$, located in general position. Let 
$\mu_{1}, \ldots, \mu_{q}$ be either positive integers or $\infty$ such that

$$
\sum_{j=1}^{q}\left(1-\frac{s}{\mu_{j}}\right)>s+1
$$

Then any holomorphic mapping $f: \mathbb{C} \rightarrow \mathbb{P}^{s}(\mathbb{C})$ that intersects $H_{j}$ with multiplicity at least $\mu_{j}$ (some of them may be $\infty$ ) for $1 \leq j \leq q$ must be constant.

Using Nochka's result from above we give the following example.

Example 2. Let $H(q)=\left\{H_{j}\right\}_{j=1}^{q}$ be defined in Example 1 with $q \geq 2 s+1$. We consider the property $\tilde{\mathcal{P}}_{H(q)}$ defined for any Riemann surface $\Sigma$ by

$$
\tilde{\mathcal{P}}_{H(q)}(\Sigma)=\left\{g \in \mathcal{H}(\Sigma): g(\Sigma) \text { is ramified over } H_{j}\right. \text { with multiplicity }
$$

at least $\mu_{j}$ (may be $\infty$ ) such that $\left.\sum_{j=1}^{q}\left(1-s / \mu_{j}\right)>s+1\right\} \cup\{$ constant maps $\}$.

We claim that it is a compact property.

Indeed, it is easy to see that $\tilde{\mathcal{P}}_{H(q)}$ satisfies trivially the axioms I) and II). As for axiom III), let $\left\{g^{(n)}\right\} \subset \tilde{\mathcal{P}}_{H(q)}(\Sigma)$ with $g^{(n)}$ locally uniformly converging to $g \in \mathcal{H}(\Sigma)$. We need to show that $g \in \tilde{\mathcal{P}}_{H(q)}(\Sigma)$. We may assume that $g$ is not constant. For each (fixed) $j$ with $1 \leq j \leq q$, we claim that $g$ intersects $H_{j}$ with multiplicity at least $\mu_{j}$. In fact, if $g(\Sigma)$ completely omits $H_{j}$ or $g(\Sigma) \subset H_{j}$, then, from the definition, the $g$ intersects $H_{j}$ with multiplicity $\infty \geq \mu_{j}$. Now let $z_{0} \in \Sigma$ with $<\mathbf{g}, \mathbf{a}_{j}>\left(z_{0}\right)=0$, where $\mathbf{g}$ is a local reduced representation (note that the property $<\mathbf{g}, \mathbf{a}_{j}>\left(z_{0}\right)=0$ is indeed independent of the choice of the reduced representation of $g$ ). We need to show that $z_{0}$ is the zero $<\mathbf{g}, \mathbf{a}_{j}>$ with order at least $\mu_{j}$. Since $g^{(n)}$ converges uniformly to $g$ around $z_{0}, g^{(n)}$ has a local reduced representation $\mathbf{g}^{(n)}$ such that $\mathbf{g}^{(n)}$ uniformly converges to a reduced representation $\mathbf{g}$ of $g$ in a neighborhood of $z_{0}$. This is true due to the following reason: we can choose a coordinate hyperplane - say, the first coordinate hyperplane $\left\{\left[w_{0}: \cdots: w_{s}\right] \mid w_{0} \neq 0\right\}$ - such that $g\left(z_{0}\right)$ is not contained in this hyperplane, and hence there is a neighborhood $U_{z_{0}}$ of $z_{0}$ such that $g\left(U_{z_{0}}\right)$ omits this hyperplane; since $g^{(n)}$ converges uniformly to $g, g^{(n)}\left(U_{z_{0}}\right)$ also omits the first coordinate hyperplane for $n \geq n_{0}$ (we may need to shrink $U_{z_{0}}$ if necessary). Thus, for $n \geq n_{0}$, we can choose the local reduced representation $\mathbf{g}^{(n)}=\left(1, g_{1}^{(n)}, \ldots, g_{s}^{(n)}\right)$ for $g^{(n)}$ and the local reduced representation $\mathbf{g}=\left(1, g_{1}, \ldots, g_{s}\right)$ for $g$ on $U_{z_{0}}$. Then, obviously, $g^{(n)}$ converges uniformly to $g$ implies that $g_{i}^{(n)}$ converges uniformly to $g_{i}$ on $U_{z_{0}}$ for each $1 \leq i \leq s$. Therefore, by the Hurwitz theorem, $<\mathbf{g}^{(n)}, \mathbf{a}_{j}>$ 
and $\left\langle\mathbf{g}, \mathbf{a}_{j}>\right.$ have the same number of zeros with counting multiplicities in a neighborhood of $z_{0}$, for $n$ big enough. Thus, $z_{0}$ is the zero of $<\mathbf{g}, \mathbf{a}_{j}>$ with order at least $\mu_{j}$ since, by the assumption, $z_{n}$ is the zero of $<\mathbf{g}^{(n)}, \mathbf{a}_{j}>$ with order at least $\mu_{j}$ and $z_{n} \rightarrow z_{0}$. This proves the claim. Hence axiom III) is verified. So $\tilde{\mathcal{P}}_{H(q)}$ is a closed property. From Nochka's result, we see that (ii) of Theorem 1 is not satisfied. Thus, (i) of Theorem 1 must satisfy, i.e., $\tilde{\mathcal{P}}_{H(q)}$ is a compact property.

\section{Minimal surfaces immersed in $\mathbb{R}^{m}$}

In this section, we apply the above results to establish a general result (see Theorem 2) about the Gauss curvature estimate for minimal surfaces in $\mathbb{R}^{m}$ whose Gauss maps satisfy certain property $\mathcal{P}$.

3.1. Preparations. In this part, we recall some results that will be used later.

We first recall the following construction result of minimal surfaces.

Proposition 3.1 ([3, 16]). Let $\Sigma$ be an open Riemann surface, and let $\omega_{1}, \omega_{2}, \ldots, \omega_{m}$ be holomorphic 1-forms on $\Sigma$ having no common zero and no real periods, and locally satisfying the identity

$$
g_{1}^{2}+g_{2}^{2}+\cdots+g_{m}^{2}=0
$$

for holomorphic functions $g_{i}$ with $\omega_{i}=g_{i} d z$. Set

$$
x_{i}=2 \operatorname{Re} \int_{z_{0}}^{z} \omega_{i},
$$

for arbitrary fixed points $z_{0}$ of $\Sigma$. Then the surface $x=\left(x_{1}, x_{2}, \cdots, x_{m}\right)$ : $\Sigma \rightarrow \mathbb{R}^{m}$ is a minimal surface immersed in $\mathbb{R}^{m}$ such that its Gauss map is the map $g=\left[\omega_{1}: \omega_{2}: \cdots: \omega_{m}\right]: \Sigma \rightarrow Q_{m-2}(\mathbb{C})$ and the induced metric is given by

$$
d s^{2}=2\left(\left|\omega_{1}\right|^{2}+\left|\omega_{2}\right|^{2}+\cdots+\left|\omega_{m}\right|^{2}\right) .
$$

The following is the general version of Hurwitz's theorem.

Lemma $3.2([\mathbf{1 7}])$. Let $f_{j}: M \rightarrow N$ be a sequence of holomorphic maps between two connected complex manifolds converging uniformly on every compact subset of $M$ to a holomorphic map $f$. If the image of each map $f_{j}$ misses a divisor $D$ of $N$, then either the image of $f$ misses $D$ or it lies entirely in $D$.

The following lemma is the well-known Ahlfors-Schwarz lemma.

Lemma $3.3([\mathbf{1 2}])$. Let $d s^{2}=\lambda^{2}(z)|d z|^{2}$ be a Hermitian pseudometric on the unit disk $D$ (i.e., $\lambda$ may have isolated zeros). If the Gauss curvature $K$ of the metric $d s^{2}$ satisfies

$$
K \leq-1
$$


then

$$
\lambda(z) \leq \frac{1}{1-|z|^{2}}
$$

In other words, the Poincare metric on $D$ is the largest among the Hermitian pseudo-metrics on the unit $D$ whose Gauss curvatures are bounded above by -1 .

Lemma 3.4 ([17], Lemma 2.1). Let $D(r)$ be the disk of radius $r$, $0<r<1$, and let $R$ be the hyperbolic radius of $D(r)$ in the unit disc. Let

$$
d s^{2}=\lambda^{2}(z)|d z|^{2}
$$

be any conformal metric on $D(r)$ with the property that the geodesic distance from $z=0$ to $|z|=r$ is greater than or equal to $R$. If the Gauss curvature $K$ of the metric $d s^{2}$ satisfies

$$
-1 \leq K \leq 0,
$$

then the distance of any point to the origin in the metric $d s^{2}$ is greater than or equal to the hyperbolic distance in terms of the Poincare metric.

Lemma 3.4 implies the following.

Lemma 3.5 ([17], Lemma 2.2). Let $d s_{n}^{2}$ be a sequence of conformal metrics on the unit disk $D$ whose curvatures satisfies $-1 \leq K_{n} \leq 0$. Suppose that $D$ is a geodesic disk of radius $R_{n}$ with respect to the metric $d s_{n}^{2}$, where $R_{n} \rightarrow \infty$, and that the metrics $d s_{n}^{2}$ converge, uniformly on compact sets, to a metric $d s^{2}$. Then all distances to the origin with respect to $d s^{2}$ are greater than or equal to the corresponding hyperbolic distances in D. In particular, $d s^{2}$ is complete.

We also need the following Yau-version of the Schwarz lemma (See [25]), which is dual to the above Ahlfors-Schwarz lemma.

Lemma $3.6([\mathbf{2 5}])$. Let $d s^{2}=\lambda^{2}(z)|d z|^{2}$ be a complete Hermitian metric on the unit disk $D$ whose Gauss curvature $K$ satisfies $K \geq-1$. Then

$$
\frac{1}{1-|z|^{2}} \leq \lambda(z)
$$

Proof. Applying Theorem 2 in [25] with $M=N=D, d s_{M}^{2}=d s^{2}$ as above, and $d s_{N}^{2}$ being the Poincare metric on $D, K_{1}=-1$ and $K_{2}=-1$, gives

$$
\frac{1}{1-|z|^{2}} \leq \lambda(z)
$$


3.2. Minimal surfaces in $\mathbb{R}^{m}$. Let $M$ be a minimal surface immersed in $\mathbb{R}^{m}$. Take an immersion $x: M \rightarrow \mathbb{R}^{m}$. Make $M$ into a Riemann surface, denoted by $\Sigma$, by decreeing that the 1 -form $d u+\sqrt{-1} d v$ is of type $(1,0)$, where $(u, v)$ are isothermal coordinates of $M$. The generalized Gauss map of the minimal surface is given by

$$
g=\left[\frac{\partial x_{1}}{\partial z}: \frac{\partial x_{2}}{\partial z}: \cdots: \frac{\partial x_{m}}{\partial z}\right]: \Sigma \rightarrow Q_{m-2}(\mathbb{C}) \subset \mathbb{P}^{m-1}(\mathbb{C}),
$$

which is a holomorphic map, where $z=u+i v$. The metric $d s^{2}$ on $\Sigma$, induced from the standard metric in $\mathbb{R}^{m}$, is $d s^{2}=\sum_{j=1}^{m}\left|\frac{\partial x_{j}}{\partial z}\right|^{2} d z d \bar{z}$, and its Gauss curvature is

$$
K=-4 \frac{\left|\mathbf{g} \wedge \mathbf{g}^{\prime}\right|^{2}}{|\mathbf{g}|^{6}}=-4 \frac{\sum_{1 \leq j<k \leq m}\left|g_{j} g_{k}^{\prime}-g_{k} g_{j}^{\prime}\right|^{2}}{\left(\sum_{j=1}^{m}\left|g_{j}\right|^{2}\right)^{3}},
$$

where $\mathbf{g}=\left(g_{1}, g_{2}, \cdots, g_{m}\right), g_{j}=\frac{\partial x_{j}}{\partial z}, 1 \leq j \leq m$, in terms of local coordinate $z$.

We also need the following lemma which play a key role in the proof.

Lemma $3.7\left([\mathbf{1 7}]\right.$, Lemma 3.2). Let $x^{(n)}=\left(x_{1}^{(n)}, x_{2}^{(n)}, \cdots, x_{m}^{(n)}\right)$ : $\Sigma \rightarrow \mathbb{R}^{m}$ be a sequence of minimal immersions, and let $g^{(n)}: \Sigma \rightarrow$ $Q_{m-2}(\mathbb{C}) \subset \mathbb{P}^{m-1}(\mathbb{C})$ be the sequence of their (generalized) Gauss maps. Suppose that $\left\{g^{(n)}\right\}$ converges uniformly on every compact subset of $\Sigma$ to a non-constant holomorphic map $g: \Sigma \rightarrow Q_{m-2}(\mathbb{C}) \subset \mathbb{P}^{m-1}(\mathbb{C})$ and that there is some $p_{0} \in \Sigma$ such that for each $j, 1 \leq j \leq m,\left\{x_{j}^{(n)}\left(p_{0}\right)\right\}$ converges. Assume also that $\left\{\left|K_{n}\right|\right\}$ is uniformly bounded, where $K_{n}$ is that Gauss curvature of the minimal surface $x^{(n)}$. Then either

(i) a subsequence $\left\{K_{n_{\ell}}\right\}$ of $\left\{K_{n}\right\}$ converges to zero, or

(ii) a subsequence $\left\{x^{\left(n_{\ell}\right)}\right\}$ of $\left\{x^{(n)}\right\}$ converges to a minimal immersion, $x: \Sigma \rightarrow \mathbb{R}^{m}$, whose Gauss map is $g$.

Definition 3.1. Let $\mathcal{P}$ be a compact property. We say that $\mathcal{P}$ satisfies a curvature estimate if there exists a positive constant $C=C(\mathcal{P})$, such that any conformal minimal immersion $x: \Sigma \rightarrow \mathbb{R}^{m}$ whose Gauss map lies in $\mathcal{P}(\Sigma)$ verifies the curvature bound

$$
|K(p)| d^{2}(p) \leq C, \quad p \in \Sigma
$$

where $K$ is the Gauss curvature of $x$ and $d(p)$ is its geodesic distance from $p$ to the boundary of $\Sigma$.

Before we go to our main theorem, we recall a theorem proved by Osserman (see [15]) concerning minimal surfaces in $\mathbb{R}^{m}$ whose Gauss map omits a neighborhood of some hyperplane in $\mathbb{P}^{m-1}(\mathbb{C})$. 
Proposition 3.8 (Osserman [15]). An inequality of the form (5) holds for all minimal surfaces in $\mathbb{R}^{m}$ whose Gauss map omits a neighborhood of some hyperplane in $\mathbb{P}^{m-1}(\mathbb{C})$.

We are now ready to prove the following general theorem about the Gauss curvature estimate, in the spirit of Bloch's heuristic principle, which is one of the main theorems in the paper.

Theorem 2. Let $\mathcal{P}$ be a compact property for holomorphic maps from Riemann surfaces into $\mathbb{P}^{m-1}$. Then the following alternative holds:

i) $\mathcal{P}$ satisfies a Gauss curvature estimate, or

ii) there is a conformal complete minimal immersion $x: D \rightarrow \mathbb{R}^{m}$ whose Gauss map lies in $\mathcal{P}(D)$ and whose Gauss curvature $K$ satisfies $|K(0)|=1$ and $|K| \leq 4$ in $D$.

Proof. Suppose the assertion in i) does not hold. We will construct a minimal surface satisfying ii).

Since, by assumption that the curvature estimate fails, there exists a sequence of (possibly non-complete) minimal surfaces $x^{(n)}: \Sigma_{n} \rightarrow \mathbb{R}^{m}$ and points $p_{n} \in \Sigma_{n}$, such that $\left|K_{n}\left(p_{n}\right)\right| d_{n}^{2}\left(p_{n}\right) \rightarrow \infty$ and such that the Gauss map $g^{(n)}$ of $x^{(n)}$ lies in $\mathcal{P}\left(\Sigma_{n}\right)$.

We claim that the surfaces $M_{n}$ parameterized by $x^{(n)}: \Sigma_{n} \rightarrow \mathbb{R}^{m}$ indeed can be chosen so that

$(6) K_{n}\left(p_{n}\right)=-1,-4 \leq K_{n} \leq 0$ on $M_{n}$ for all $n$ and $d_{n}\left(p_{n}\right) \rightarrow \infty$.

We now prove the claim. First, by changing the Riemann surface $\Sigma_{n}$ if necessary, we can assume that $M_{n}$ is a geodesic disc centered at $p_{n}$. Let $M_{n}^{\prime}=\left\{p \in M_{n}: d_{n}\left(p, p_{n}\right) \leq d_{n}\left(p_{n}\right) / 2\right\}$. Then $K_{n}$ is uniformly bounded on $M_{n}^{\prime}$ and $d_{n}^{\prime}(p)=$ distance of $p$ to the boundary of $M_{n}^{\prime}$ tends to zero as $p \rightarrow \partial M_{n}^{\prime}$. Hence $\left|K_{n}(p)\right|\left(d_{n}^{\prime}(p)\right)^{2}$ has a maximum at a point $p_{n}^{\prime}$ interior to $M_{n}^{\prime}$. Therefore,

$$
\left|K_{n}\left(p_{n}^{\prime}\right)\right| d_{n}^{\prime}\left(p_{n}^{\prime}\right)^{2} \geq\left|K_{n}\left(p_{n}\right)\right| d_{n}^{\prime}\left(p_{n}\right)^{2}=\frac{1}{4}\left|K_{n}\left(p_{n}\right)\right| d_{n}^{2}\left(p_{n}\right) \rightarrow \infty .
$$

So we can replace $M_{n}$ by $M_{n}^{\prime}$, with $\left|K_{n}\left(p_{n}^{\prime}\right)\right| d_{n}^{\prime}\left(p_{n}^{\prime}\right)^{2} \rightarrow \infty$. We then rescale $M_{n}^{\prime}$ to make $K_{n}\left(p_{n}^{\prime}\right)=-1$. By the invariance under scaling of the quantity $K(p) d(p)^{2}$, we will have $d_{n}^{\prime}\left(p_{n}^{\prime}\right) \rightarrow \infty$; here, without causing confusion, we use the same notation $d_{n}^{\prime}$ to denote the geodesic distance with respect to the rescaled metric. Again we can assume that $M_{n}^{\prime}$ is a geodesic disc centered at $p_{n}^{\prime}$, and we let

$$
M_{n}^{\prime \prime}=\left\{p \in M_{n}^{\prime} \mid d_{n}\left(p, p_{n}^{\prime}\right)<\frac{d_{n}^{\prime}\left(p_{n}^{\prime}\right)}{2}\right\} .
$$

Then $p \in M_{n}^{\prime \prime}$ implies that $d_{n}^{\prime}(p) \geq \frac{d_{n}^{\prime}\left(p_{n}^{\prime}\right)}{2}$ and

$$
\left|K_{n}(p)\right| \frac{d_{n}^{\prime}\left(p_{n}^{\prime}\right)^{2}}{4} \leq\left|K_{n}(p)\right| d_{n}^{\prime}(p)^{2} \leq\left|K_{n}\left(p_{n}^{\prime}\right)\right| d_{n}^{\prime}\left(p_{n}^{\prime}\right)^{2}=d_{n}^{\prime}\left(p_{n}^{\prime}\right)^{2} .
$$


Therefore, $\left|K_{n}(p)\right| \leq 4$ on $M_{n}^{\prime \prime}$. Furthermore, $d_{n}^{\prime \prime}\left(p_{n}^{\prime}\right)=d\left(p_{n}^{\prime}, \partial M_{n}^{\prime \prime}\right)=$ $d_{n}^{\prime}\left(p_{n}^{\prime}\right) / 2 \rightarrow \infty$. This proves the claim.

By translation of $\mathbb{R}^{m}$, we can assume that $x^{(n)}\left(p_{n}\right)=0$. We can also assume that $\Sigma_{n}$ is, by taking its universal covering if necessary, simply connected. By the uniformization theorem, $\Sigma_{n}$ is conformally equivalent to either the unit disc $D$ or the complex plane $\mathbb{C}$, and we can suppose that $p_{n}$ maps onto 0 for each $n$. But the latter case that $\Sigma_{n}$ is conformally equivalent to $\mathbb{C}$ is impossible because by Lemma $2.2 \mathrm{~g}^{(n)}$ is constant, so $K_{n} \equiv 0$, which contradicts the condition that $\left|K_{n}(0)\right|=1$. So we have constructed a sequence of minimal surfaces, $x^{(n)}: D \rightarrow \mathbb{R}^{m}$, that satisfies (11) and $g^{(n)} \in \mathcal{P}(D)$. Since $\mathcal{P}$ is a compact property, by IV), there exist a subsequence of $\left\{g^{(n)}\right\}$ (without loss of generality we still denote it $\left\{g^{(n)}\right\}$ ), such that $g^{(n)}$ converges uniformly on every compact subset of $D$ to a map $g \in \mathcal{H}(D)$. Since compact property implies closed property, we have $g \in \mathcal{P}(D)$.

Now, we claim that $g$ is non-constant. If this is not the case, we may assume that $g$ is a constant map, and $g$ maps $D$ onto a single point $P$. Let $H$ be any hyperplane not containing $P$, and let $U, V$ be disjoint neighborhoods of $H$ and $P$, respectively. Let $C$ be the constant in Proposition 3.8, such that

$$
|K(p)|^{1 / 2} d(p) \leq C
$$

for any minimal surface $S$ in $\mathbb{R}^{m}$ whose Gauss map omits the neighborhood $U$ of $\mathrm{H}$, where $p$ is a point of $S$ and $d(p)$ is the geodesic distance of $p$ to the boundary of $S$. Choose $r<1$ such that the hyperbolic distance $R$ of $z=0$ to $|z|=r$ satisfies $R>C$. Since $g^{(n)}$ converges uniformly to $g$ on $\bar{D}(r)$, the image of $|z|=r$ lies in the neighborhood $V$ of $P$ for sufficiently large $n$-say, $n \geq n_{0}$. It follows that for any $n \geq n_{0}$, the image of $\bar{D}(r)$ under $g^{(n)}$ omits the neighborhood $U$ of $H$ and we may therefore apply the above inequality to conclude

$$
\left|K_{n}(0)\right|^{1 / 2} d_{n}(r) \leq C,
$$

where $d_{n}(r)$ is the geodesic distance from $z=0$ to the boundary of the surface $x^{(n)}: D(r) \rightarrow \mathbb{R}^{m}$. But $\left|K_{n}(0)\right|=1$ for all $n$, and hence $d_{n}(r) \leq C$ for $n \geq n_{0}$. On the other hand, the surface $x^{(n)}: D \rightarrow \mathbb{R}^{m}$ is a geodesic disk of radius $R_{n}$. If we reparametrize by $w=r_{n} z$, where the subset $\left\{w:|w|<r_{n}\right\}$ has hyperbolic radius $R_{n}$, then the circle $|z|=r$ corresponds to $|w|=r_{n} r$, and by Lemma 3.4 the distance in the surface metric from $z=0$ to any point on $|z|=r$, or equivalently, $|w|=r_{n} r$, is greater than or equal to the hyperbolic distance from $z=0$ to $|w|=r_{n} r$. Letting $n \rightarrow \infty$, we have $R_{n} \rightarrow \infty$ and $r_{n} \rightarrow 1$, so the hyperbolic radius of $|w|=r_{n} r$ tends to the hyperbolic radius of $|w|=r$, which is $R$. Since by assumption $R>C$, we have for $n$ sufficiently large that the 
surface distance from $z=0$ to $|z|=r$ is greater than $C$, contradicting $d_{n}(r) \leq C$. Thus, we conclude that $g$ is non-constant.

Therefore, the hypotheses of Lemma 3.7 are satisfied. Since $\left|K_{n}(0)\right|=$ 1 , the possibility (i) of Lemma 3.7 cannot happen. Thus, a subsequence $\left\{x^{\left(n_{\ell}\right)}\right\}$ of $\left\{x^{(n)}\right\}$ converges to a minimal immersion $x: D \rightarrow \mathbb{R}^{m}$, whose Gauss map is $g$. By (6) and Lemma 3.5, $x$ is complete. This complete the proof the Theorem 2 .

q.e.d.

Theorem 2 gives the following consequence.

Theorem 3. Let $\mathcal{P}$ be a compact property. Then the following assertions are equivalent:

(i) $\mathcal{P}$ satisfies the curvature estimate.

(ii) The plane is the only complete minimal surface in $\mathbb{R}^{m}$, whose Gauss map satisfies $\mathcal{P}$.

(iii) There are not complete minimal surfaces, conformally equivalent to the disc, with bounded Gauss curvature and whose Gauss map lies in $\mathcal{P}(D)$.

Proof. Obviously, we have i) $\Longrightarrow$ ii) $\Longrightarrow$ iii), and from Theorem 2 we conclude that iii) $\Longrightarrow \mathrm{i}$ ).

q.e.d.

3.3. Some consequences of Theorem 2. We now derive some consequences of Theorem 2. We recall the first concrete in the previous section (Example 1): Denote by $H(q)=\left\{H_{j}\right\}_{j=1}^{q}$ a set of hyperplanes in $\mathbb{P}^{m-1}(\mathbb{C})$, located in general position. We consider the property $\mathcal{P}_{H(q)}$ defined for any Riemann surface $\Sigma$ by

$$
\begin{aligned}
\mathcal{P}_{H(q)}(\Sigma)= & \left\{g \in \mathcal{H}(\Sigma): \text { either } g(\Sigma) \cap H_{j}=\emptyset \text { or } g(\Sigma) \subset H_{j}\right. \\
& \text { for every } 1 \leq j \leq q\} \cup\{\text { constant maps }\}
\end{aligned}
$$

Above, we showed that if $q \geq 2(m-1)+1$, then it is a compact property. We also need the following result of $\mathrm{Ru}([\mathbf{2 2}])$.

Proposition 3.9 ([22], Theorem 1). Let $M$ be a non-flat complete minimal surface immersed in $\mathbb{R}^{m}$ and assume that the Gauss map $G$ of $M$ is $k$, non-degenerate $(0 \leq k \leq m-1)$. Then $G$ can omit at most $(k+1)(m-k / 2-1)+m$ hyperplanes in $\mathbb{P}^{m-1}(\mathbb{C})$ located in general position.

Thus, Theorem 2, together with Ru's above result, and the fact that $\mathcal{P}_{H(q)}$ is a compact property for $q \geq 2 m-1$, gives the following result, which is the main result of Osserman and Ru ([17]) (see Theorem 1.1 in [17]) with slight modification.

Theorem A ([17, Theorem 1.1],). Let $M$ be a minimal surface immersed in $\mathbb{R}^{m}$. If there are $q>m(m+1) / 2$ hyperplanes $H_{j}$ in $\mathbb{P}^{m-1}(\mathbb{C})$, located in general position, such that the generalized Gauss map $g$ of $M$ 
satisfies $g(M) \cap H_{j}=\emptyset$ or $g(M) \subset H_{j}$, for each $1 \leq j \leq q$, then there exists a constant $C>0$, such that

$$
|K(p)| d^{2}(p) \leq C
$$

for any $p \in M$, where $K$ is the Gauss curvature of $x$ and $d$ is its geodesic distance to the boundary of $M$.

Proof. From Theorem 2, we only need to show that if $x: D \rightarrow \mathbb{R}^{m}$ is a conformal complete minimal immersion, whose Gauss map $g$ satisfies either $g(D) \cap H_{j}=\emptyset$ or $g(D) \subset H_{j}$, for each $1 \leq j \leq q$ with $q>$ $m(m+1) / 2$, then $g$ must be constant, since in this case (ii) in Theorem 2 does not hold, because $K \equiv 0$; thus, (i) must hold, i.e., $\mathcal{P}_{H(q)}$ satisfies a Gauss curvature estimate.

To prove it, denote by $J \subset\{1, \ldots, q\}$ such that $j \in J$ if and only if $g(D) \subset H_{j}$, and let $X_{J}:=\bigcap_{j \in J} H_{j}$. We can identify $X_{J}$ with a project space of dimension $m-1-k$, where $k=\# J$. Notice that the restrictions of $H_{j}, j \notin J$, to $X_{J}$ are hyperplanes that are still in general position in $X_{J}$. Note that $q-k>m(m+1) / 2-k \geq(m-k)(m-k+1) / 2$; it follows from Proposition 3.9 that $g$ is constant. This completes the proof of Theorem A.

q.e.d.

Note that Theorem A, in the complete case, gives the following result, which is the main result of $\mathrm{Ru}([\mathbf{2 2}])$ with slight modification.

Theorem B ([22]). Let $M$ be a complete minimal surface immersed in $\mathbb{R}^{m}$. If there are $q>m(m+1) / 2$ hyperplanes $H_{j}$ in $\mathbb{P}^{m-1}(\mathbb{C})$, located in general position, such that the generalized Gauss map $g$ of $M$ satisfies $g(M) \cap H_{j}=\emptyset$ or $g(M) \subset H_{j}$, for each $1 \leq j \leq q$, then $M$ must be flat.

Next, we use the second concrete example in the previous section. Let $H(q)=\left\{H_{j}\right\}_{j=1}^{q}$ be a set of hyperplanes in $\mathbb{P}^{m-1}(\mathbb{C})$, located in general position. Assume that $q \geq 2 m-1$. We consider the property $\mathcal{P}_{H(q), 2 m-1}$ that, for any Riemann surface $\Sigma$ and for $g \in \mathcal{H}\left(\Sigma, \mathbb{P}^{m-1}\right)$, $g \in \mathcal{P}_{H(q), 2 m-1}(\Sigma)$ if and only if either $g$ is a constant map or $g$ is ramified over $H_{j}$ with multiplicity at least $\mu_{j}($ may be $\infty)$ with

$$
\sum_{j=1}^{q}\left(1-\frac{m-1}{\mu_{j}}\right)>m .
$$

We showed in the second example above that it is a compact property.

We also need the following result of $\mathrm{Ru}$ (see $[\mathbf{2 3}]$ ).

Proposition 3.10 ([23], Theorem 1). Let $M$ be a complete minimal surface immersed in $\mathbb{R}^{m}$. If its Gauss map is ramified over a set of hyperplanes $H_{j}, 1 \leq j \leq q$, in $\mathbb{P}^{m-1}(\mathbb{C})$ located in general position 
multiplicity at least $\mu_{j}$ (some of them may be $\infty$ ) with

$$
\sum_{j=1}^{q}\left(1-\frac{m-1}{\mu_{j}}\right)>\frac{(m+1) m}{2}
$$

then $M$ must be a plane.

Thus, Theorem 2, together with Ru's above result, as well as the fact that $\mathcal{P}_{H(q), 2 m-1}$ is a compact property for $q \geq 2 m-1$, gives the following result.

Theorem 4. Let $M$ be a minimal surface immersed in $\mathbb{R}^{m}$. If its Gauss map is ramified over a set of hyperplanes $H_{j}, 1 \leq j \leq q$ in $\mathbb{P}^{m-1}(\mathbb{C})$ located in general position multiplicity at least $\mu_{j}$ (some of them may be $\infty$ ) with

$$
\sum_{j=1}^{q}\left(1-\frac{m-1}{\mu_{j}}\right)>\frac{(m+1) m}{2},
$$

then there exists a constant $C>0$, such that

$$
|K(p)| d^{2}(p) \leq C
$$

for any $p \in M$, where $K$ is the Gauss curvature of $x$ and $d$ is its geodesic distance to the boundary of $M$.

\section{The use of the boundness $|K| \leq 4$ of the Gauss curvature $K$}

Recall that, from Theorem 2 under the assumption that $\mathcal{P}$ is a compact property, if $\mathcal{P}$ does not satisfy a curvature estimate (i.e., (i) in Theorem 2 does not hold), then one comes up a non-flat complete minimal surface in $\mathbb{R}^{m}$ with bounded curvature $|K| \leq 4$ (i.e., (ii) in Theorem 2 holds). In this section, we show that this additional information of $|K| \leq 4$ helps to greatly simplify the proof of the known results. This demonstrates again the importance of Theorem 2 (which is one of our main results in the paper).

A simpler proof of Theorem $B$ (as well as Theorem A): we give here a simpler proof of Theorem B (which is the main result of [22]) with the following statement. Let $M$ be a complete minimal surface immersed in $\mathbb{R}^{m}$. If there are $q>m(m+1) / 2$ hyperplanes $H_{j}$ in $\mathbb{P}^{m-1}(\mathbb{C})$, located in general position, such that the generalized Gauss map $g$ of $M$ satisfies $g(M) \cap H_{j}=\emptyset$ or $g(M) \subset H_{j}$, for each $1 \leq j \leq q$, then $M$ must be flat.

Here is the proof: If the Gauss curvature estimate

$$
|K(p)| d^{2}(p) \leq C
$$

holds, then we are done since the condition that $M$ is complete implies that $K \equiv 0$ and thus $M$ is flat. So we can assume the Gauss curvature 
estimates fails. Then (ii) in Theorem 2 holds, i.e., there is a (new) nonflat complete minimal surface immersed in $\mathbb{R}^{m}$-we denote it by $x$ : $D \longrightarrow \mathbb{R}^{m}$ - such that its Gauss curvature satisfies

$$
K(0)=-1 \quad \text { and }|K(z)| \leq 4, z \in D
$$

and its Gauss map $G$ satisfies $G(D) \cap H_{j}=\emptyset$ or $G(D) \subset H_{j}$, for each $1 \leq j \leq q$, where $H_{j}$ are $q$ hyperplanes in $\mathbb{P}^{m-1}(\mathbb{C})$, located in general position with $q>\frac{(m+1) m}{2}$. We now use this additional condition $|K(z)| \leq 4$ to finish the proof.

Denote by $J \subset\{1, \ldots, q\}$ such that $j \in J$ if and only if $G(D) \subset$ $H_{j}$, and let $X_{J}:=\bigcap_{j \in J} H_{j}$ and $\# J=\ell$. We can identify $X_{J}$ with a project space of dimension $m-1-\ell$. Without loss of generality, we may assume that $X_{J}=\mathbb{P}^{n}(\mathbb{C})$, where $n=m-1-\ell$, i.e., $G: D \rightarrow$ $\mathbb{P}^{n}(\mathbb{C})$. Notice that the restrictions of $H_{j}, j \notin J$, to $X_{J}$ (which we still denote it by $H_{j}$ ) are hyperplanes that are still in general position in $X_{J}$. Since $G: D \rightarrow \mathbb{P}^{n}(\mathbb{C})$ is non-constant, we can assume that $G(D)$ is contained in a $k$-dimensional linear subspace of $\mathbb{P}^{n}(\mathbb{C})$, but not a lower one, with $0<k \leq n$. In other words, $G: M \rightarrow \mathbb{P}^{k}(\mathbb{C}) \subset \mathbb{P}^{n}(\mathbb{C})$ is linearly non-degenerate. Since $H_{1}, H_{2}, \ldots, H_{t}$ are in general position in $\mathbb{P}^{n}(\mathbb{C})$, where $t=q-\ell$, their restrictions (we still denote them by $\left.H_{1}, \ldots, H_{t}\right)$ are in $n$-subgenernal position in $\mathbb{P}^{k}(\mathbb{C})$. Denote by $\omega(j)$ the Nochka weights associated to the hyperplanes $H_{1}, \ldots, H_{t}$. From [22], the Nochka weights satisfy

$$
t-2 n+k-1=\theta\left(\sum_{j=1}^{t} \omega(j)-k-1\right)
$$

for some number $\theta>0$ with

$$
0<\omega(j) \theta<1
$$

for all $1 \leq j \leq t$, and

$$
1 \leq \frac{n-1}{k+1} \leq \theta \leq \frac{2 n-k+1}{k+1} .
$$

We recall the following (see the main lemma in $[\mathbf{2 2}]$ ).

Lemma 4.1. Let $H_{1}, \ldots, H_{t}$ be hyperplanes in $\mathbb{P}^{k}(\mathbb{C})$ in $n$-subgeneral position, and let $\omega(j)$ be their Nochka weights. Let $f=\left[f_{0}: \cdots\right.$ : $\left.f_{k}\right]: D(R) \rightarrow \mathbb{P}^{k}(\mathbb{C})$ be a non-degenerate holomorphic map whose image omits these hyperplanes. Let $F=\left(f_{0}, \ldots, f_{k}\right): D(R) \rightarrow \mathbb{C}^{k+1} \backslash\{0\}$ be a reduced representation of $f$. Assume that $t>2 n-k+1$ and

$$
\frac{2 t}{N}<\frac{\sum_{j=1}^{t} \omega(j)-(k+1)}{k(k+2)} \text {. }
$$


Then there exists some positive constant $C$ such that

$$
|F|^{S} \frac{\prod_{p=0}^{k-1} \prod_{j=1}^{t}\left|F_{p}\left(H_{j}\right)\right|^{\frac{4}{N}}\left|F_{k}\right|^{1+\frac{2 t}{N}}}{\prod_{j=1}^{t}\left|F\left(H_{j}\right)\right|^{\omega(j)}} \leq C\left(\frac{2 R}{R^{2}-|z|^{2}}\right)^{\frac{k(k+1)}{2}+\sum_{p=0}^{k-1}(k-p)^{2} \frac{2 t}{N}}
$$

where $S=\sum_{j=1}^{t} \omega(j)-(k+1)-\left(k^{2}+2 k-1\right) \frac{2 t}{N}$ and $\mathbb{P}\left(F_{p}\right)$ is the pth associated map of $f$.

Let $\tilde{G}=\left(g_{0}, \cdots, g_{k}\right)$ be a reduced representation of $G$; then the metric $d s^{2}$ on $\Sigma$ induced from the standard metric on $\mathbb{R}^{m}$ is given by

$$
d s^{2}=2|\tilde{G}|^{2}|d z|^{2} \text {. }
$$

From $-4 \leq K \leq 0$ and Yau's version of the Schwarz Lemma (See Lemma 3.6, above),

$$
\frac{1}{4\left(1-|z|^{2}\right)} \leq|\tilde{G}|
$$

On the other hand, let $S=\sum_{j=1}^{t} \omega(j)-(k+1)-\left(k^{2}+2 k-1\right) 2 t / N$ as in Lemma 4.1, and let

$$
\begin{aligned}
\delta & =S-\left[k(k+1) / 2+\sum_{p=0}^{k-1}(k-p)^{2} 2 t / N\right] \\
& =\sum_{j=1}^{t} \omega(j)-(k+1)-\frac{k(k+1)}{2}-\left[\left(k^{2}+2 k-1\right)+\sum_{p=0}^{k-1}(k-p)^{2}\right] \frac{2 t}{N} .
\end{aligned}
$$

From the condition that $q>\frac{m(m+1)}{2}$ and by using (7) and (9), we have

$$
\begin{aligned}
& \theta\left\{\sum_{j=1}^{t} \omega(j)-(k+1)-\frac{k(k+1)}{2}\right\}=t-2 n+k-1-\theta \frac{k(k+1)}{2} \\
\geq & t-2 n+k-1-\frac{k(2 n-k+1)}{2} \\
= & q-\ell-2(m-1-\ell)+k-1-\frac{k(2(m-1-\ell)-k+1)}{2} \\
= & q-2 m+k+1-\frac{k(2 m-k-1)}{2}+(k+1) \ell \\
> & \frac{m(m+1)}{2}-(2 m-k-1) \frac{k+2}{2} \geq 0
\end{aligned}
$$


for any integer $k$ with $1 \leq k \leq n \leq m-1$. Hence, we can choose $N$ such that

$$
\frac{4+4 t\left[k^{2}+2 k-1+\sum_{p=0}^{k}(k-p)^{2}\right]}{\sum_{j=1}^{t} \omega(j)-(k+1)-k(k+1) / 2}>N>\frac{2 t\left[k^{2}+2 k-1+\sum_{p=0}^{k}(k-p)^{2}\right]}{\sum_{j=1}^{t} \omega(j)-(k+1)-k(k+1) / 2} .
$$

so that

$$
\delta>0, \text { and } \delta_{0}:=\frac{4}{\delta N}>1
$$

Write hyperplanes $H_{j}, j=1, \ldots, t$, as

$$
H_{j}=\left\{\left[z_{0}: \cdots: z_{k}\right]: a_{j, 0} z_{0}+\cdots+a_{j, k} z_{k}=0\right\}
$$

Then, by the condition that $G$ is linearly non-degenerate, for each pair $(j, p)$ with $1 \leq p \leq k$, there is $i_{1}, \ldots, i_{p}$ such that

$$
\psi_{j, p}=\sum_{\ell \neq i_{1}, \cdots, i_{p}} a_{j, \ell} W\left(g_{\ell}, g_{i_{1}}, \cdots, g_{i_{p}}\right)
$$

does not vanish identically. Every $\psi_{j, p}$ is holomorphic, so they have only isolated zeros. From Lemma 4.1, notice that by the definition of $\psi_{j, p}(w)$ we have $\left|\psi_{j, p}\right|<\left|\tilde{G}_{p}\left(H_{j}\right)\right|$,

$$
|\tilde{G}|^{S} \frac{\prod_{p=0}^{k-1} \prod_{j=1}^{t}\left|\psi_{j, p}\right|^{4 / N}\left|\tilde{G}_{k}\right|^{1+2 t / N}}{\prod_{j=1}^{t}\left|\tilde{G}\left(H_{j}\right)\right|^{\omega(j)}} \leq C\left(\frac{2}{1-|z|^{2}}\right)^{k(k+1) / 2+\sum_{p=0}^{k-1}(k-p)^{2} 2 t / N}
$$

where $S=\sum_{j=1}^{t} \omega(j)-(k+1)-\left(k^{2}+2 k-1\right) 2 t / N$. Combing (10) and (11) implies that

$$
\begin{aligned}
\frac{1}{1-|z|^{2}} \leq 4|\tilde{G}| \leq C\left(\frac{\prod_{j=1}^{t}\left|\tilde{G}\left(H_{j}\right)\right|^{\omega(j)}}{\left|\tilde{G}_{k}\right|^{1+2 t / N} \prod_{p=0}^{k-1} \prod_{j=1}^{t}\left|\psi_{j, p}\right|^{4 / N}}\right)^{\frac{1}{S-\left[k(k+1) / 2+\sum_{p=0}^{k-1}(k-p)^{2} 2 t / N\right]}} \\
=C \lambda
\end{aligned}
$$


for some positive constant $C>0$, where

$$
\begin{aligned}
\lambda:= & \left(\frac{\prod_{j=1}^{t}\left|\tilde{G}\left(H_{j}\right)\right|^{\omega(j)}}{\left|\tilde{G}_{k}\right|^{1+2 t / N} \prod_{p=0}^{k-1} \prod_{j=1}^{t}\left|\psi_{j, p}\right|^{4 / N}}\right)^{\frac{1}{S-\left[k(k+1) / 2+\sum_{p=0}^{k-1}(k-p)^{2} 2 t / N\right]}} \\
& =\left(\frac{\prod_{j=1}^{t}\left|\tilde{G}\left(H_{j}\right)\right|^{\omega(j)}}{\left|\tilde{G}_{k}\right|^{1+2 t / N} \prod_{p=0}^{k-1} \prod_{j=1}^{t}\left|\psi_{j, p}\right|^{4 / N}}\right)^{\frac{1}{\delta}}
\end{aligned}
$$

We now consider the new metric

$$
d s_{0}^{2}=\lambda^{2}|d z|^{2}
$$

on $D^{\prime}=D \backslash\left(\left\{\tilde{G}_{k}=0\right\} \bigcup \bigcup_{j, p}\left\{z: \psi_{j, p}(z)=0\right.\right.$ or $\left.\left.\psi_{j, p}(1 / z)=0\right\}\right)$, with $\lambda$ being as above. Then it is easily to see that $d s_{0}^{2}$ is flat, since $\psi_{j, p}$ and $\tilde{G}_{k}$ are holomorphic. We claim that it is complete at the punctures. Indeed, if either $\tilde{G}_{k}\left(z_{0}\right)=0$ or $\psi_{j, p}\left(z_{0}\right)=0$ for some positive $p$ and $j$, then it is easy to see that

$$
\left|d s_{0}\right| \sim \frac{c}{\left|z-z_{0}\right|^{\delta_{0}}}|d z|
$$

around $z_{0}$, where $c>0, \delta_{0}=\frac{4}{\delta N}>1$. Therefore, $d s_{0}^{2}$ is complete on $\left\{\tilde{G}_{k}=0\right\} \bigcup \bigcup_{j, p}\left\{z: \psi_{j, p}(z)=0\right.$ or $\left.\psi_{j, p}(1 / z)=0\right\}$. Moreover, $\frac{1}{C\left(1-|z|^{2}\right)} \leq$ $\lambda(z)$ from $(12)$, so $d s_{0}^{2}$ is complete at the boundary of the disc. So the universal cover of $D^{\prime}$ must be $\mathbb{C}$ and this contradiction proves Theorem B.

\section{References}

[1] A. Bloch, La conception actuelle de la théorie des fonctions entières et méromorphes, Enseignement math. 25 (1926), 83-103, JFM 52.0315.03

[2] M. do Carmo \& C.K. Peng, Stable minimal surfaces in $\mathbb{R}^{3}$ are planes, Bull. Amer. Math. Soc. 1 (1979), 903-906, MR 0546314, Zbl 0442.53013.

[3] S.S. Chern \& R. Osserman, Complete minimal surfaces in euclidean n-space, J. Anal. Math. 19 (1967), 15-34, MR 0226514, Zbl 0172.22802.

[4] A. Eremenko \& M. Sodin, Value distribution of meromorphic functions and meromorphic curves from the point of view of potential theory, St. Petersburg Math. J. 3 (1992), 109-136, MR 1120844, Zbl 0791.30028.

[5] D. Fisher-Colbrie \& R. Schoen, The structure of complete stable minimal surfaces in 3-manifolds of non-negative scalar curvature, Comm. Pure. Appl. Math. 33 (1980), 199-211, MR 0562550, Zbl 0439.53060. 
[6] H. Fujimoto, On the number of exceptional values of the Gauss map of minimal surfaces, J. Math. Soc. Japan 40 (1988), 235-247, MR 0930599, Zbl 0629.53011.

[7] H. Fujimoto, On the Gauss curvature of minimal surfaces, J. Math. Soc. Japan 44 (1992), 427-439, MR 1167375, Zbl 0759.53004.

[8] H. Fujimoto, Value distribution theory of the Gauss map of minimal surfaces in $\mathbb{R}^{m}$, Aspects of Mathematics E21, Friedr. Vieweg \& Teubner Verlag, 1993, MR 1218173, Zbl 1107.32004.

[9] M. Green, The hyperbolicity of the complement of $2 n+1$ hyperplanes in general position in $\mathbb{P}^{n}$ and related results, Proc. Amer. Math. Soc. 66 (1977), 109-113, MR 0457790, Zbl 0366.32013.

[10] E. Heinz, Über die Lösungen der Minimalflächengleichung, Nachr. Akad. Wiss. Göttingen., Phys. K1. II (1952), 51-56, MR 0054182, Zbl 0048.15401.

[11] D. Hoffman \& R. Osserman, The geometry of the generalized Gauss map, Mem. Amer. Math. Soc. 236 (1980), 1-105, MR 0587748, Zbl 0469.53004.

[12] S. Lang, Introduction to complex hyperbolic space, Springer, Berlin, 1987, MR 0886677, Zbl 0628.32001.

[13] E.I. Nochka, On the theory of meromorphic functions, Soviet Math. Dokl 27 (1983), 377-381, MR 0701289, Zbl 0552.32024.

[14] J. Noguchi \& J. Winkelmann, Holomorphic curves and integral points off divisors, Math. Z. 239 (2002), 593-610, MR 1893854, Zbl 1011.32012.

[15] R. Osserman, Global properties of Minimal Surfaces in $E^{3}$ and $E^{n}$, Ann. of Math. 80 (1964), 340-364, MR 0179701, Zbl 0134.38502.

[16] R. Osserman, A survey of minimal surfaces, 2nd edition, Dover Publ. Inc., New York, 1986, MR 0852409, Zbl 0209.52901.

[17] R. Osserman \& M. Ru, An estimate for the Gauss curvature of minimal surfaces in $\mathbb{R}^{m}$ whose Gauss map omits a set of hyperplanes, J. Differential Geom. 48 (1997), 578-593, MR 1484891, Zbl 0918.53003.

[18] X.C. Pang, Bloch's principle and normal criterion, Sci. China, Ser. A, 32 (1989), 782-791, MR 1057999, Zbl 0687.30023.

[19] X.C. Pang, On normal criterion of meromorphic functions, Sci. China, Ser. A, 33 (1990), 521-527, MR 1070538, Zbl 0706.30024.

[20] A.V. Pogorelov, On the stability of minimal surfaces, Soviet Math. Dokl. 24 (1981), 274-276, MR 0630142, Zbl 0495.53005.

[21] A. Ros, The Gauss map of minimal surfaces, Differential Geometry, Valencia 2001, Proceedings of the conference in honour of Antonio M. Naveira, edited by O. Gil-Medrano and V. Miquel, World Scientific, (2002) 235-252, MR 1922054, Zbl 1028.53008.

[22] M. Ru, On the Gauss map of minimal surfaces immersed in $\mathbb{R}^{n}$, J. Differential Geom. 34 (1991) 411-423, MR 1131437, Zbl 0733.53005.

[23] M. Ru, Gauss map of minimal surfaces with ramification, Tran. Amer. Math. Soc. 339 (1993), 751-764, MR 1191614, Zbl 0792.53003.

[24] R. Schoen, Estimates for stable minimal surfaces in three-dimensional manifolds, Ann. of Math. Studies, Vol. 103 Princeton University Press, Princeton, NJ (1983), 111-125, MR 0795231, Zbl 0532.53042.

[25] S.T. Yau, A general Schwarz lemma for Kähler manifolds, Amer. J. of Math. 1 (1978), 197-203, MR 0486659, Zbl 0424.53040. 
[26] L. Zalcman, A heuristic principle in complex function theory, Amer. Math. Monthly 82 (1975), 813-817, MR 0379852, Zbl 0315.30036.

[27] L. Zalcman, Normal families: New perspectives, Bull. Amer. Math. Soc. (N.S.) 35 (1998), 215-230, MR 1624862, Zbl 1037. 30021.

Department of Mathematics University of Shanghai for Science and Technology

Shanghai 200093, P.R. China

E-mail address: Xiaojunliu2007@hotmail.com

Department of Mathematics East China Normal University

Shanghai 200241, P.R.China

E-mail address: xcpang@math.ecnu.edu.cn 